\title{
In vivo confocal microscopy classification in the diagnosis of meibomian gland dysfunction
}

\author{
Matthieu Randon $\mathbb{1}^{1,2} \cdot$ Vittoria Aragno ${ }^{1,2} \cdot$ Rachid Abbas $^{3} \cdot$ Hong Liang $^{1,4} \cdot$ Antoine Labbé $^{1,2,4} \cdot$ \\ Christophe Baudouin ${ }^{1,2,4}$
}

Received: 16 May 2018 / Revised: 28 August 2018 / Accepted: 5 October 2018 / Published online: 12 December 2018

(c) The Royal College of Ophthalmologists 2018

\begin{abstract}
Aim Meibomian gland dysfunction (MGD) is one of the most common disorders in ophthalmology. The aim of this study was to evaluate the use of this in vivo confocal microscopy (IVCM)-MGD description to classify patients affected by clinical MGD and measure the correlation with standard clinical criteria and subjective symptoms.

Methods One hundred eyes of 100 patients suffering from MGD and 15 eyes of normal subjects were included. A comprehensive evaluation with the ocular surface disease index (OSDI), Schirmer test, tear break-up time (TBUT), tear osmolarity, Oxford score, Meibomian gland expression, palpebral IVCM, and meibography was performed. Then each patient was classified using a new IVCM classification: type 0 for normality, type 1 for meibum obstruction, type 2 for inflammation, and type 3 for fibrosis.

Results The mean age of patients was $52 \pm 20$ years old, the OSDI was $38 \pm 23$, the BUT $5 \pm 2.6 \mathrm{~s}$, the Schirmer test $13 \pm 7 \mathrm{~mm}$, tear osmolarity $300 \pm 11 \mathrm{osmol} / \mathrm{L}$, the Oxford score $0.5 \pm 0.6$, the meibum expression score $1.7 \pm 1.02$, and the meibography score $1.3 \pm 0.9$. The IVCM MG classification of the 15 normal subjects was 0. For MGD patients, $29 \%$ were in type 1, 40\% were type 2, and 31\% were type 3 . The patients in IVCM MG type 2 had a higher OSDI $(p=0.001)$ compared with the other types. There was a strong correlation between the IVCM score and the meibography score $(r=0.71$ $p<0.0001)$.

Conclusion This new IVCM classification provided a practical pathophysiological system for MGD. By giving objective criteria, this IVCM classification may help advance the understanding of patients' symptoms and enhance treatment effectiveness in MGD.
\end{abstract}

Christophe Baudouin

cbaudouin@15-20.fr

1 Department of Ophthalmology III, Quinze-Vingts National Ophthalmology Hospital, DHU Sight Restore, INSERM-DHOS CIC, Paris, France

2 Department of Ophthalmology, Ambroise Paré Hospital, APHP, University of Versailles Saint-Quentin-en-Yvelines, Versailles, France

3 Department of Biostatistics and Epidemiology, Gustave Roussy, Villejuif, France; INSERM U1018, CESP, Université Paris-Sud, Université Paris-Saclay, Villejuif, France

4 Sorbonne Universités, UPMC Univ Paris 06, INSERM, CNRS, Institut de la Vision, 17 rue Moreau, 75012 Paris, France

\section{Introduction}

Meibomian gland dysfunction (MGD) is a chronic, diffuse abnormality of the meibomian glands, commonly characterized by terminal duct obstruction and/or qualitative/ quantitative changes in glandular secretion [1]. MGD is one of the most common disorders encountered in clinical practice, with a reported prevalence around $38-77 \%[2,3]$. The pathogenesis of MGD is based on meibum secretion changes and solidification that can lead to gland obstruction. This situation can evolve to an inflammatory stage. Over time, this chronic condition, if not interrupted, may result in gland atrophy. This statement is a continuous hypothesis and the meibomian gland (MG) inflammation or fibrosis could be primitive. The clinical consequences of this gland dysfunction are impaired tear film secretion, tear film instability, corneal damage, and symptoms of eye irritation [4]. MGD is considered a major cause of dry 
eye with both hyperevaporative and hyposecretory mechanisms [5].

In clinical practice, MG and tear film evaluations are commonly based on slit lamp examination. The ophthalmological assessment is comprehensive of tear film break-up time (TBUT), corneal fluorescein staining, MG macroscopic morphology and expression, and the Schirmer test. Subjective symptoms are collected using appropriate questionnaires, the most often used being the ocular surface disease index (OSDI) and the SPEED score [1].

Various diagnostic tools have been proposed to better investigate MGD, such as meibography, anterior-segment OCT (AS-OCT), and in vivo confocal microscopy (IVCM) [6, 7].

Meibography, based on MG transillumination, is a useful tool to observe meibomian glands objectively $[1,6,7]$. Several studies have found a good correlation between MG loss (MGL) evaluated with meibography and meibum quality [8], OSDI, lipid layer pattern, noninvasive BUT and age [9]. Moreover, it has been demonstrated that the MGL is a good parameter to differentiate MGD patients from healthy subjects [10]. However, this technique cannot observe inflammation within or around the MGs.

AS-OCT, using a longer wavelength in comparison to meibography, presents a transversal view of the MG. It is possible to obtain more detailed images and identify the deeper layers of the MG [11]. Like meibography, AS-OCT [12] provides only morphological data at a macroscopic scale, without real visualization of the meibum or the inflammatory infiltration implicated in MGD pathology. Moreover, meibography alone is not sufficient for the diagnosis of MGD, but should be interpreted in association with other clinical parameters [13].

In vivo confocal microscopy (IVCM) is a non-invasive imaging technique that gives qualitative images of the ocular surface and eyelids, including MG, with histology-like resolution. Recently, a new IVCM score has been proposed to classify MGD, based on the evaluation of three main parameters: meibum reflectivity, intraepithelial and interglandular inflammation, and fibrosis [14]. Four types based on MG morphology were defined by our group [14]: type 0 is the absence of MGD, type 1 is the obstructive disease, type 2 is the inflammatory disease, and type 3 is the fibrosis state. Even if different types can be found in the same subject, the classification is based on the most widespread pattern on IVCM images. The aim of this study was to further evaluate the use of this new IVCM-MGD description to classify patients affected by clinical MGD and measure the correlation with standard clinical criteria and subjective symptoms.

\section{Methods}

\section{Subjects}

This prospective single-center study was conducted at the Investigation Centre (CIC INSERM 1423) of the QuinzeVingts Hospital. The study was conducted in accordance with the Declaration of Helsinki (1964) and approved by our Ethics Committee CPP-Ile de France (number 10793).

One hundred eyes of 100 patients with MGD and 15 eyes of 15 healthy subjects were enrolled. All subjects were informed and accepted the ophthalmic evaluation. The criteria for the diagnosis of MGD were OSDI>13, signs of posterior blepharitis (edema, erythema, and telangiectasia of the lid margin) and abnormal meibum expression. We excluded patients with blepharitis therapy other than artificial tears at the moment of the examination. Fifteen subjects without blepharitis, OSDI $\leq 13$ and normal meibum expression were enrolled. The most affected eye, in terms of signs of posterior blepharitis and meibum expression, was studied. For the healthy subjects, the right eye was chosen.

\section{Ophthalmological evaluation}

All patients underwent an ophthalmological assessment that consisted of OSDI, tear film osmolarity (TearScience Inc, Morrisville, NC, USA), Schirmer test type I, TBUT, corneal staining with fluorescein $0.5 \%$ (Oxford score) and meibum expression. Meibomian gland secretion was graded from 0 to 3 according to Shimazaki et al. grading $(0=$ clear secretion, 1 = cloudy meibum expressed with mild pressure, 2 = cloudy meibum expressed with more than moderate pressure, $3=$ toothpaste secretion or no expressible secretion) [15].

The infrared meibography was performed with the Oculus Keratograph $5 \mathrm{M}^{\circledR}$ (Wetzlar, Germany) to evaluate the meibomian gland morphology. Images of meibomian glands in the entire lower eyelids were obtained. The meibography scores were graded according to the extent of the meibomian gland dropout as described in our previous study [16]. It was graded from 0 to $3(0=$ no gland loss; $1=$ loss $<33 \%, 2=$ loss between 33 and $66 \%, 3=$ loss $>66 \%$ ) as shown in Fig. 1 .

\section{In vivo laser confocal microscopy}

In vivo laser confocal microscopy (Heidelberg RetinaTomograph II-Rostock Cornea Module: HRTII-RCM; Heidelberg Engineering $\mathrm{GmbH}$, Dossenheim, Germany) was performed on the most affected eye for all subjects after topical anesthesia with $0.4 \%$ oxybuprocaine. The laser source employed by the HRTII-RCM was a diode laser with a 670-nm wavelength. Two-dimensional images, covering a 
Fig. 1 MGD staging with meibography: a stage 0 , no gland loss; b stage 1, loss $<33 \%$; c stage 2 , loss between 33 and $66 \%$; d stage 3 , loss $>66 \%$
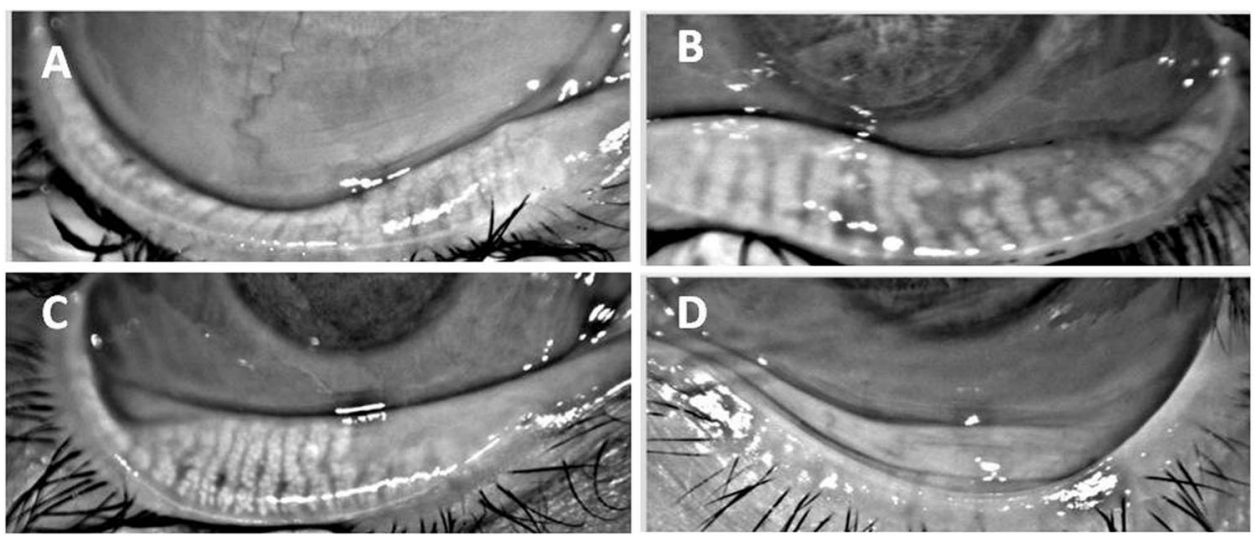

$400 \times 400-\mu \mathrm{m}$ area, were obtained. When the first superficial conjunctival cells were visualized, the pachymetry was set at zero and the focal plane was moved to reach the subconjunctival tissue where the MGs could be visualized. MGs were scanned from the nasal to temporal eyelid section moving horizontally. The following parameters were analyzed and classified (classification adapted from Villani [16]). The combination of grades of meibum reflectivity, intraepithelial/interglandular inflammation, and glandular fibrosis made it possible to determine the MG type. Type 0 was a normal MG; type 1 was the obstructed MG with a heterogeneous meibum reflectivity without inflammation or fibrosis; type 2 was the inflammation of $\mathrm{MG}$ without fibrosis; type 3 was the fibrosis of $\mathrm{MG}$ with or without meibum heterogeneity and with or without inflammation. Overlapping of two types was a possibility in some subjects, so it was decided that the major type was retained.

The MG classification is summarized in Table 1 and Fig. 2. A typical session lasted between 5 and $8 \mathrm{~min}$. Two operators (VA and MR) performed the IVCM examinations. We acquired as many scans of the lower eyelid as we could without exceeding more than 8 min of examination duration. All images were reviewed, and the five bestquality images were selected for analysis. Images were assessed in a masked way by one observer (MR). The mean value of the five images was recorded for each parameter.

\section{Statistical analyses}

Analyses were done on all patients included. Demographic and clinical characteristics for the individuals with MGD and those without MGD diagnosis were reported as mean \pm standard deviation (or median and interquartile intervals) for quantitative data, and frequencies and proportions for categorical data. They were compared with the Wilcoxon rank-sum for continuous data and $\chi^{2}$ - or Fisher tests for categorical data. All analyses were done on SAS 9.4 (SAS Institute, Inc., Cary, NC, USA).
Table 1 MGD type system based on MG-IVCM analysis

\begin{tabular}{lllll}
\hline & Type 0 & Type 1 & Type 2 & Type 3 \\
Grade M & 0 & $1-3$ & $0-3$ & $0-3$ \\
\hline
\end{tabular}

Grade 0: homogeneous hyporeflective meibum

Grade 1: some focal

heterogeneous reflectivity

Grade 2: multiple

heterogeneous reflectivity

Grade 3: very heterogeneous

reflectivity [17]

\begin{tabular}{lllll}
\hline Grade I & 0 & $0-1$ & $2-3$ & $0-3$ \\
\hline
\end{tabular}

Grade 0: no hyperreflective points

Grade 1: some intraepithelial hyperreflective points $<300 / \mathrm{mm}^{2}$

Grade 2: numerous intraepithelial hyperreflective points $>300 / \mathrm{mm}^{2}$

Grade 3: numerous intraepithelial and interglandular points [14]

\begin{tabular}{lllll}
\hline Grade F & 0 & 0 & $0-1$ & 2 \\
\hline
\end{tabular}

Grade 0: normal epithelium

Grade 1: mild fibrosis

Grade 2: severe fibrosis

\section{Results}

\section{Descriptive analysis}

In the overall population including the 100 MGD patients and 15 normal subjects, the mean age was $52 \pm 20$ years, there were 64 women $(56 \%)$, the OSDI was $38 \pm 23$, the BUT $5 \pm 2.6 \mathrm{~s}$, the Schirmer test $13 \pm 7 \mathrm{~mm}$, the tear osmolarity $300 \pm 11 \mathrm{osmol} / \mathrm{L}$, the Oxford grade $0.5 \pm 0.6$, the meibum expression score $1.7 \pm 1.02$ and the meibography score $1.3 \pm 0.9$. 

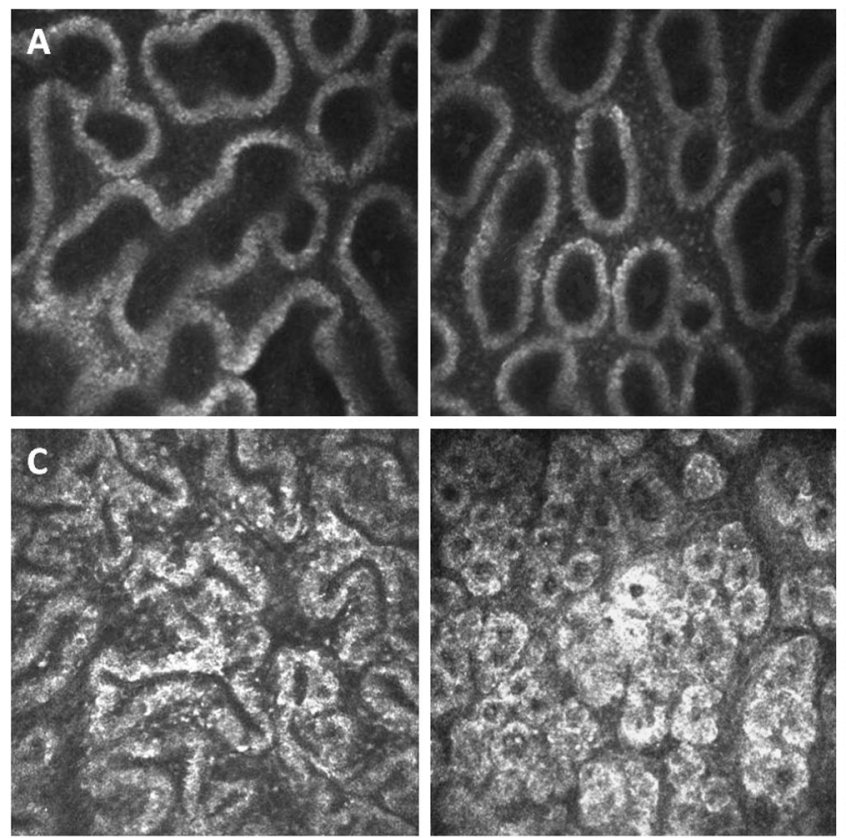

Fig. 2 MGD staging analyzed with in vivo confocal microscopy (IVCM). a type 0 in IVCM, normal meibomian glands; b type 1 in IVCM, lumen obstruction with minimal inflammation; c type 2 in

This IVCM score classified the 15 normal subjects to type 0 with no mistakes (with normal MG), and within the group of MGD patients, 29 patients (29\%) were categorized to type 1 (with alteration of meibum), 40 patients (40\%) to type 2 (association of altered meibum and inflammation), and 31 patients (31\%) to type 3 (MG fibrosis) (Table 2).

Of the 115 patients, the meiboscore classified 15 normal subjects and 11 patients (23\%) to score $0 ; 44$ patients (38\%) to score $1 ; 29$ patients $(25 \%)$ to score 2 and 16 patients (14\%) to score 3.

Type 0 subjects had no symptoms or clinical signs. There were $47 \%$ women with a mean age of $29.7 \pm 7$ years. The mean OSDI and osmolarity were $6 \pm 3$ and $288 \pm 7 \mathrm{mOsm} / \mathrm{L}$, respectively. The mean Schirmer I test and TBUT were $23 \pm$ $5 \mathrm{~mm}$ and $9.3 \pm 0.8 \mathrm{~s}$, respectively. Infrared meibography and the Oxford score were 0 for all normal subjects.

In the group of subjects with IVCM MG type 1, there were $65 \%$ women with a mean age of $44 \pm 20$ years. The mean OSDI and osmolarity were $37 \pm 18$ and $300 \pm 9$ $\mathrm{mOsm} / \mathrm{L}$, respectively. The mean Schirmer I test, TBUT and Oxford test were $14 \pm 7 \mathrm{~mm}, 5.2 \pm 2.6 \mathrm{~s}$, and $0.31 \pm 0.47$, respectively. The mean meibum expression and infrared meibography score were $2.10 \pm 0.94$ and $0.76 \pm 0.64$, respectively.

Among the subjects with IVCM MG type 2, 57\% were women and the mean age was $57 \pm 16$ years. The mean OSDI and osmolarity were $51 \pm 20$ and $305 \pm 11 \mathrm{mOsm} / \mathrm{L}$, respectively. The mean Schirmer I test, TBUT and Oxford test were $10.9 \pm 7 \mathrm{~mm}, \quad 3.73 \pm 2 \mathrm{~s}$, and $0.60 \pm 0.63$,
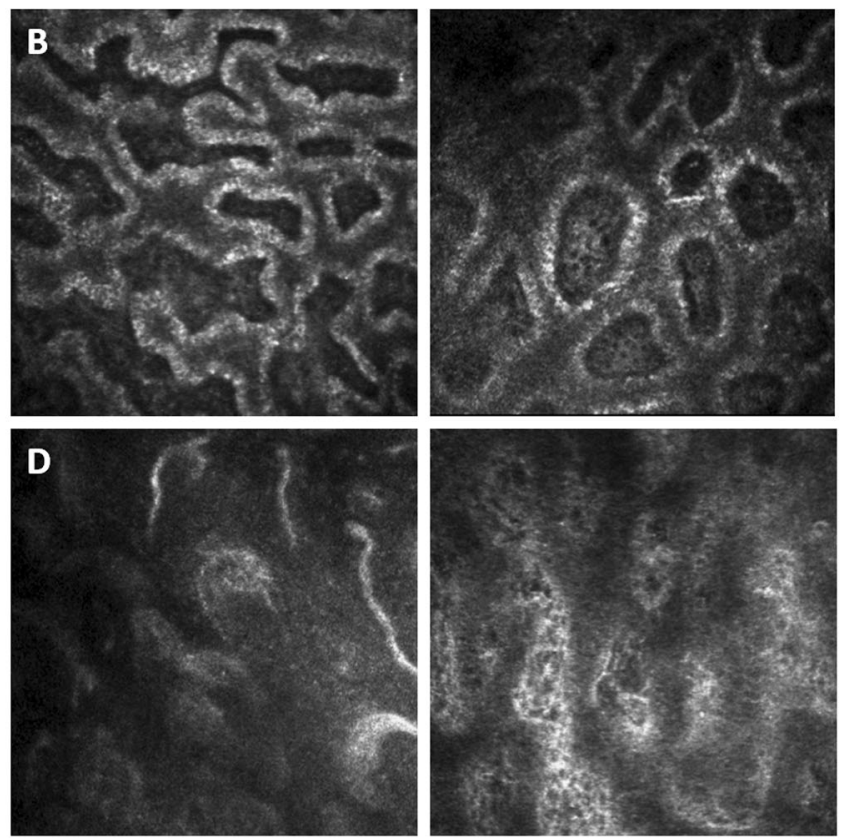

IVCM, intraepithelial, and interglandular inflammation; d, type 3 in IVCM, fibrosis with epithelium destruction

respectively. The mean meibum expression and meibography score were $1.78 \pm 0.73$ and $1.53 \pm 0.78$, respectively.

Among the subjects with IVCM MG type 3,48\% were women and the mean age was $65 \pm 12$ years. The mean OSDI and osmolarity were $39 \pm 18$ and $301 \pm 9 \mathrm{mOsm} / \mathrm{L}$, respectively. The mean Schirmer I test, TBUT and Oxford test were $12 \pm 7 \mathrm{~mm}, 4.7 \pm 1.9 \mathrm{~s}$, and $0.68 \pm 0.70$, respectively. The mean meibum expression and meibography score were $2.23 \pm 0.76$ and $2.13 \pm 0.76$, respectively.

\section{Correlation analysis}

The IVCM score and the meibography score were strongly correlated $(r=0.71 P<0.0001$ [95\% CI 0.61-0.79]). In IVCM MG type $1,35 \%, 55 \%$, and $10 \%$ of the patients had a meibography score of 0,1 , and 2 , respectively. In IVCM MG type 2, 5\%,50\%, 33\%, and $12 \%$ of patients had a meibography score of $0,1,2$, and 3 , respectively. In IVCM MGD type 3,23\%, 42\%, and 35\% of the patients had a meibography score of 1,2 , and 3 , respectively.

The correlation between the IVCM score and the meibum expression score was also significant $(r=0.40, p<0.0001$ [95\% CI, 0.23-0.54]). In IVCM MGD type 1, 45\% of patients had a meibum expression score of 1; in IVCM MGD type 2, $57 \%$ had a meibum expression score of 2; in IVCM MGD type $3,42 \%$ had a meibum expression score of 3 .

Correlations between IVCM, tear osmolarity and the Oxford score were also significant $(0.30$ and 0.34 , respectively; $p<0.05$ for both correlations). Similarly, the IVCM 
Table 2 Demographic and clinical data analyses in terms of IVCM type

\begin{tabular}{|c|c|c|c|c|c|}
\hline & \multicolumn{5}{|c|}{ IVCM-MGD type } \\
\hline & 0 & 1 & 2 & 3 & $P^{*}$ \\
\hline Number of eyes & 15 & 29 & 40 & 31 & \\
\hline Age $($ mean \pm SD) & $29 \pm 7$ & $44 \pm 20$ & $57 \pm 16$ & $\begin{array}{l}65 \pm \\
12\end{array}$ & $0.5^{*}$ \\
\hline Sex (\%women) & 47 & 65 & 57 & 48 & $0.2 * *$ \\
\hline OSDI $($ mean $\pm \mathrm{SD})$ & $6 \pm 3$ & $37 \pm 18$ & $51 \pm 20$ & $\begin{array}{l}39 \pm \\
18\end{array}$ & $0.001 *$ \\
\hline Oxford score $(\%)$ & & & & & $0.05 * *$ \\
\hline Score 0 & 100 & 69 & 47.5 & 45 & \\
\hline Score 1 & 0 & 31 & 45 & 42 & \\
\hline Score 2 & 0 & 0 & 7.5 & 13 & \\
\hline $\begin{array}{l}\text { Osmolarity (mean } \pm \\
\mathrm{SD}, \mathrm{mOsm} / \mathrm{L})\end{array}$ & $\begin{array}{l}288 \pm \\
7\end{array}$ & $300 \pm 9$ & $305 \pm 11$ & $\begin{array}{l}301 \pm \\
9\end{array}$ & $0.4^{*}$ \\
\hline $\begin{array}{l}\text { Schirmer I test } \\
(\text { mean } \pm \mathrm{SD}, \mathrm{mm})\end{array}$ & $23 \pm 5$ & $14 \pm 7$ & $10.9 \pm 7$ & $12 \pm 7$ & $0.05 *$ \\
\hline TBUT $($ mean $\pm \mathrm{SD}, \mathrm{s})$ & $\begin{array}{l}9.3 \pm \\
0.8\end{array}$ & $5.2 \pm 2$ & $3.7 \pm 2$ & $\begin{array}{l}4.7 \pm \\
1.9\end{array}$ & $0.05 *$ \\
\hline $\begin{array}{l}\text { Meibomian } \\
\text { expression }(\%)\end{array}$ & & & & & $0.001 * *$ \\
\hline Stage 0 & 100 & 3 & 5 & 0 & \\
\hline Stage 1 & 0 & 28 & 25 & 19 & \\
\hline Stage 2 & 0 & 24 & 57 & 39 & \\
\hline Stage 3 & 0 & 45 & 13 & 42 & \\
\hline $\begin{array}{l}\text { Infrared meibography } \\
\text { score }(\%)\end{array}$ & & & & & $0.001 * *$ \\
\hline Score 0 & 100 & 35 & 5 & 0 & \\
\hline Score 1 & 0 & 55 & 50 & 23 & \\
\hline Score 2 & 0 & 100 & 33 & 42 & \\
\hline Score 3 & 0 & & 12 & 35 & \\
\hline
\end{tabular}

SD standard deviation

$* \chi^{2}$ or Fisher exact test

$* *$ Wilcoxon rank-sum test

score was correlated with TBUT and the Schirmer test $(-0.36$ and -0.31 , respectively, $p<0.05$ for both correlations). Seven patients had a grade 2 Oxford score. Among those patients, three had a type 2 IVCM MGD score and four had a type 3 IVCM MGD score. The OSDI was not correlated with the IVCM MGD score. The OSDI was higher in IVCM type 2 (51) as compared to types 1 and 3 (37 and 39 , respectively, $p=0.001$ ).

\section{Discussion}

In the last few years, the role of MGD as the leading cause of dry eye disease has been emphasized [1, 13]. Recently, a new approach for understanding MGD pathophysiology has been proposed by our group [5, 17]. A vicious circle has been postulated, starting with meibum stasis and meibomian gland blockage, followed by bacterial proliferation and inflammation, release of lipases and esterases, the consequent increase in meibum melting temperature and finally gland fibrosis and atrophy. This statement is a continuous hypothesis and usually the patient kept the same features over time.

The difficulties of the dry eye diagnosis are characterized by a lack of correlation between symptoms and objective clinical findings [1]. Routine clinical tests are probably not able to detect important signs that could explicate symptoms reported from most patients such as pain, irritation, itching, and blurred vision. Therefore, we need a more precise imaging tool to help clinicians in their analysis.

We recently described an IVCM evaluation score of MGD based on four types of MG examinations: type 0 corresponds to normal MG, type 1 to the obstructive state, type 2 to the inflammatory condition, and type 3 to gland fibrosis [14]. Compared with the complex potential of IVCM MG measurements, this is a semi-quantified score, which could be rapidly used by clinicians without any sophisticated imaging analysis software. We believe that inflammation plays a major role and that its assessment is highly important in diagnosing patient disease and assessing severity levels to drive adapted therapeutic procedures more efficiently.

In this study, we wished to validate the IVCM MG description in a larger cohort of patients and correlate it with clinical criteria, subjective symptoms, and the meibography score.

The first use of IVCM in MG was by Kobayashi et al. in 2005 [18] on normal subjects. Other studies then analyzed MG in pathological situations, such as glaucoma [19], graft versus host disease [20], atopic keratoconjunctivitis [21], contact lens wearers [22], and Demodex infestation [23, 24]. Previous studies have already proposed several parameters to classify meibomian gland alterations and Villani et al. provide support for the occurrence of primary, age-related MG atrophy [25]. The bland MGD corresponds to the obstructive MGD and the inflammatory meibomitis is equivalent to the inflammation type MGD. Messmer EM et al. did not describe the fibrotic type [23]. Matsumoto et al. suggested acinar unit diameter and density as useful parameters to evaluate MG changes in MGD [26]. Ibrahim et al. demonstrated the efficacy, sensitivity, and specificity of meibomian gland acinar longest diameter (MGALD), MG acinar shortest diameter (MGASD), inflammatory cell density (ICD) and MG acinar unit density (MGAUD) in the diagnosis of MGD [21]. Nevertheless, these parameters are not easy to collect during examination, because they require a post-exam evaluation and analysis of the acquired images with sophisticated software. For these reasons, in clinical practice, they cannot be adopted as easily achievable 
parameters to decide, manage, and evaluate therapy for MGD. Moreover, we think that dilation is not mandatory for meibum obstruction type. We hypothesized that meibum reflectivity is a more sensible sign for meibum obstruction.

Moreover, we thought that defining the different types of MG based on the description of the lumen, epithelium, and the space between acini was more useful given that it corresponded more closely to the MGD vicious circle. Our classification was demonstrated to be mildly correlated with all the clinical tests: tear osmolarity, Oxford score, TBUT, Schirmer test. It means that all MGD types would lead to ocular surface disease and clinical tests abnormalities. Moreover, clinical tests were not statistically different between the three types of MGD. Only the OSDI was significantly higher in type 2 . That could indicate that the only way to differentiate MGD patients and understand their painful symptomatology is a microscopic investigation with IVCM to address inflammation. In type 2, the inflammatory stage of the disease, bacterial proliferation, esterase and lipase activation, and release of inflammatory mediators probably mean more severe symptoms.

Interestingly, when MG changes are mild, i.e., type 1 of our classification, patients are less symptomatic. In these cases, IVCM is the only tool able to detect the first histological changes so that early treatment can eventually be started. In fact, in type 1 patients, there is often a very mild gland loss evidenced by meibography. In type 1 , the ocular surface system still seems able to compensate MG changes. On the contrary, in a few cases, when all the tests are normal except the OSDI, MG IVCM is able to detect the MGD.

Moreover, our classification was also correlated with the infrared meibography score, which exclusively explores gland loss [27]. Meibography does not examine the gland itself but the presence of meibum [27]. We noticed that using IVCM, the images showed obstruction, inflammation or mild fibrosis while meibography produced a similar image regardless of MG type. In fact, meibography only detects MG presence or loss and is interesting for advanced or severe MG fibrosis.

Adequate classification of patients in different types is useful not only for a pathophysiological approach and to understand patients' symptoms, but also to choose an appropriate therapy, especially a MG-targeted treatment.

The international workshop on MGD proposed four stages of disease evaluating symptoms, corneal staining and meibum expressibility [28]. Based on these stages, patient education, lid margin hygiene, tetracycline, and steroids are proposed. According to our description, the therapy could be more targeted to patients with early MG changes, especially when inflammation can be detected. Patients in type 1 would benefit from lid hygiene, LipiFlow $^{\circledR}$ or intense pulsed-light treatment to overcome meibum stasis and gland obstruction. Patients in type 2 should be treated with tetracycline and steroids. For patients in type 3, these procedures are probably not useful and lacrimal substitutes should be preferred.

This study has a number of limitations: first IVCM explores only the terminal part of the inferior tarsus with a $400 \times 400-\mu \mathrm{m}$ field, whereas meibography is able to explore the entire superior and inferior tarsus. We do not know if the images would be the same in the superior tarsus or in the proximal part of the inferior tarsus. We believe that the MG changes begin at the terminal part of the gland and then progress to the proximal part, so the IVCM should detect the first MG alterations.

Second, this is not a prospective study, so we cannot confirm whether MGD evolves from type 1 to type 2 and 3 or if a medical treatment could prevent this progression and eventually restore a normal MG pattern. We noted that during the IVCM image analysis, the MG type was generally homogenous in the different parts of the inferior eyelid, even if a few cases with overlapping types were found.

In conclusion, we have reported a large study on MGD using an IVCM-based meibomian gland description to classify different types of disease. The palpebral IVCM could help the clinician better understand patients' symptomatology when clinical signs are not correlated with symptoms.

\section{Summary}

\section{What was known before}

- The MGD is usually explored by meibography which only detects fibrosis of the meibomian gland.

\section{What this study adds}

- We herein report the classification of the meibomian gland disorder, which includes a comparison with the meiboscore. We described three different pathologies: the obstructed gland, the gland's inflammation and the gland's fibrosis.

\section{Compliance with ethical standards}

Conflict of interest The authors declare that they have no conflict of interest.

\section{References}

1. Schaumberg DA, Nichols JJ, Papas EB, et al. The international workshop on meibomian gland dysfunction: report of the subcommittee on the epidemiology of, and associated risk factors for MGD. Invest Ophthalomol Vis Sci. 2011;52:1994-2005. 
2. Hom MM, Martinson JR, Knapp LL, et al. Prevalence of meibomian gland dysfunction. Optom Vis Sci. 1990;67:710-2.

3. Molinari JF, Stanek S. Meibomian gland status comparison between active duty personnel and U.S. veterans. Mil Med. 2000;165:591-3.

4. McCulley JP, Shine WE. Meibomian gland function and the tear lipid layer. Ocul Surf. 2003;1:97-106.

5. Baudouin C. Revisiting meibomian gland dysfunction. J Fr Ophtalmol. 2014;37:757-62.

6. Ban Y, Shimazaki-Den S, Tsubota K, et al. Morphological evaluation of Meibomian glands using noncontact infrared meibography. Ocul Surf. 2013;11:47-53.

7. Geerling G, Baudouin C, Aragona P, et al. Emerging strategies for the diagnosis and treatment of meibomian gland dysfunction: Proceedings of the OCEAN group meeting. Ocul Surf. 2017;172: 179-92.

8. Eom Y, Choi KE, Kang SY, et al. Comparison of meibomian gland loss and expressed meibum grade between the upper and lower eyelids in patients with obstructive meibomian gland dysfunction cornea. Cornea. 2014;33:448-52.

9. Pult H, Riede-Pult BH. Non-contact meibography: keep it simple but effective. Cont Lens Anterior Eye. 2011;35:77-80.

10. Srinivasan S, Menzies K, Sorbara L, et al. Infrared imaging of meibomian gland structure using a novel keratograph. Optom Vis Sci. 2012;89:788-94.

11. Yoo YS, Na KS, Kim DY, et al. Morphological evaluation for diagnosis of dry eye related to meibomian gland dysfunction. Exp Eye Res. 2017;10:72-7.

12. Liang Q, Pan Z, Zhou M, et al. Evaluation of optical coherence tomography meibography in patients with obstructive meibomian gland dysfunction. Cornea. 2015;34:1193-9.

13. Wolffsohn JS. Arita R2, Chalmers R. TFOS DEWS II Diagnostic Methodology report. Ocul Surf. 2017;15:539-74.

14. Randon $M$, Liang $H$, Abbas $R$, et al. A new classification for meibomian gland diseases with in vivo confocal microscopy. J Fr Ophtalmol. 2016;39:239-47.

15. Shimazaki J, Goto E, Ono M, et al. Meibomian gland dysfunction in patients with Sjögren syndrome. Ophthalmology. 1998;105: $1485-8$.

16. Villani E, Beretta S, De Capitani M, et al. In vivo confocal microscopy of meibomian glands in Sjogren's Syndrome. Invest Ophthalomol Vis Sci. 2011;52:933-9.
17. Baudouin C, Messmer EM, Aragona P, et al. Revisiting the vicious circle of dry eye disease: a focus on the pathophysiology of meibomian gland dysfunction. Br J Opthalmol. 2016;100: $300-6$.

18. Kobayashi A, Yoshita T, Sugiyama K. In vivo findings of the bulbar/palpebral conjunctiva and presumed meibomian glands by Laser scanning confocal microscopy. Cornea. 2005;24: 985-8.

19. Agnifili L, Fasanella V, Costagliola C, et al. In vivo confocal microscopy of meibomian glands in glaucoma. Br J Opthalmol. 2013;97:343-9.

20. Ban Y, Ogawa Y, Ibrahim OM, et al. Morphologic evaluation of meibomian glands in chronic graft versus-host disease using in vivo laser confocal microscopy. Mol Vis. 2011;17: 2533-43.

21. Ibrahim OM, Matsumoto Y, Dogru M, et al. In vivo confocal microscopy evaluation of meibomian gland dysfunction in atopic-keratoconjunctivitis patients. Ophthalmology. 2012;119: 1961-8.

22. Villani E, Ceresara G, Beretta S, et al. In vivo confocal microscopy of meibomian glands in contact lens wearers. Invest Ophthalomol Vis Sci. 2011;52:5215-9.

23. Messmer EM, Torres Suarez E, Mackert MI, et al. In vivo confocal microscopy in blepharitis. Klin Monbl Augenheikd. 2005;222:894-900.

24. Randon M, Liang H, El Hamdaoui M, et al. In vivo confocal microscopy as a novel and reliable tool for the diagnosis of Demodex eyelid infestation. Br J Ophthalmol. 2015;99:336-41.

25. Villani E, Canton V, Magnani $F$, et al. The aging meibomian gland: an in vivo confocal study. Invest Ophthalmol Vis Sci. 2013;54:4735-40.

26. Matsumoto Y, Sato EA, Ibrahim OM, et al. The application of in vivo laser confocal microscopy to the diagnosis and evaluation of meibomian gland dysfunction. Mol Vis. 2008;14:1263-71.

27. Finis D, Ackermann P, Pischel N, et al. Evaluation of meibomian gland dysfunction and local distribution of meibomian gland atrophy by non-contact infrared meibography. Curr Eye Res. 2015;40:982-9.

28. Gerling G, Tauber J, Baudouin C, et al. The international Workshop on Meibomian Gland Dysfunction: report of the subcommittee on management and treatment of MGD. Invest Ophthalmol Vis Sci. 2011;52:2050-64. 